\title{
Capsaicin, a TRPV1 Ligand, Suppresses Bone Resorption by Inhibiting the Prostaglandin E Production of Osteoblasts, and Attenuates the Inflammatory Bone Loss Induced by Lipopolysaccharide
}

\author{
Megumi Kobayashi, ${ }^{1}$ Kenta Watanabe, ${ }^{1}$ Satoshi Yokoyama, ${ }^{1}$ Chiho Matsumoto, ${ }^{2}$ \\ Michiko Hirata, ${ }^{1}$ Tsukasa Tominari, ${ }^{2}$ Masaki Inada, ${ }^{1}$ and Chisato Miyaura ${ }^{1,2}$ \\ ${ }^{1}$ Department of Biotechnology and Life Science, Tokyo University of Agriculture and Technology, 2-24-16 Nakamachi, \\ Koganei, Tokyo 184-8588, Japan \\ ${ }^{2}$ Cooperative Major in Advanced Health Science, Tokyo University of Agriculture and Technology, 2-24-16 Nakamachi, \\ Koganei, Tokyo 184-8588, Japan
}

Correspondence should be addressed to Chisato Miyaura, miyaura@cc.tuat.ac.jp

Received 14 December 2011; Accepted 18 January 2012

Academic Editors: M. Brunner, M. L. Ferrandiz, G. Froldi, and T. W. Stone

Copyright ( $(2012$ Megumi Kobayashi et al. This is an open access article distributed under the Creative Commons Attribution License, which permits unrestricted use, distribution, and reproduction in any medium, provided the original work is properly cited.

\begin{abstract}
Capsaicin, a transient receptor potential vanilloid type 1 (TRPV1) ligand, regulates nerve-related pain-sensitive signals, inflammation, and cancer growth. Capsaicin suppresses interleukin-1-induced osteoclast differentiation, but its roles in bone tissues and bone diseases are not known. This study examined the effects of capsaicin on inflammatory bone resorption and prostaglandin E (PGE) production induced by lipopolysaccharide (LPS) in vitro and on bone mass in LPS-treated mice in vivo. Capsaicin suppressed osteoclast formation, bone resorption, and PGE production induced by LPS in vitro. Capsaicin suppressed the expression of cyclooxygenase-2 (COX-2) and membrane-bound PGE synthase-1 (mPGES-1) mRNAs and PGE production induced by LPS in osteoblasts. Capsaicin may suppress PGE production by inhibiting the expression of COX-2 and mPGES- 1 in osteoblasts and LPSinduced bone resorption by TRPV1 signals because osteoblasts express TRPV1. LPS treatment markedly induced bone loss in the femur in mice, and capsaicin significantly restored the inflammatory bone loss induced by LPS in mice. TRPV1 ligands like capsaicin may therefore be potentially useful as clinical drugs targeting bone diseases associated with inflammatory bone resorption.
\end{abstract}

\section{Introduction}

Bone remodeling is regulated by osteoclastic bone resorption and new bone formation by osteoblasts. Prostaglandin $\mathrm{E}_{2}$ $\left(\mathrm{PGE}_{2}\right)$ is mainly produced by osteoblasts during bone resorption associated with inflammation and acts as a potent stimulator of bone resorption [1]. Inflammatory cytokines, such as interleukin (IL)-1 and tumor necrosis factor $\alpha$ (TNF $\alpha$ ) which induces $\mathrm{PGE}_{2}$ production by osteoblasts, and $\mathrm{PGE}_{2}$ are able to induce the expression of receptor activator of NF- $\kappa \mathrm{B}$ ligand (RANKL) on the surface of osteoblasts. Two types of cyclooxygenase (COX), COX-1 and COX-2, are expressed in osteoblasts, and the expression of COX-2 is markedly induced by inflammatory stimulants. The blockage of $\mathrm{PGE}_{2}$ synthesis by nonsteroidal anti-inflammatory drugs (NSAIDs) could suppress RANKL-dependent osteoclastic bone resorption associated with inflammation $[2,3]$. Membrane-bound PGE synthase-1 (mPGES-1) is also essential for $\mathrm{PGE}_{2}$ synthesis in osteoblasts and PGE-mediated osteoclast differentiation [2, 3]. An mPGES-1-null mouse demonstrated that $\mathrm{PGE}_{2}$ production by osteoblasts is essential for inflammatory bone loss induced by lipopolysaccharide (LPS) in vivo [4].

The transient receptor potential (TRP) subfamily of ion channels contains six members (TRPV1-TRPV6), most of which were thermal-sensitive cation channels. TRPV1 has 
been cloned from a cDNA library of the dorsal root ganglion in sensory neurons, and the TRPV1 signal is suggested to be a potential regulator of pain associated with thermal stimulation $[5,6]$. Capsaicin is a natural ligand for TRPV1 that regulates nerve-related pain-sensitive signals, inflammation, and cancer growth [7]. Mouse osteoblasts express TRPV1, and capsaicin suppresses IL-1-induced osteoclast differentiation in the cocultures of mouse bone marrow cells and osteoblasts [8]. Rossi et al. [9] reported that human preosteoclasts express TRPV1 and its signal modulates RANKLmediated osteoclast differentiation. Recent studies suggesting some of the possible roles of TRPV1 in bone metabolism are controversial, and the physiological and pharmacological effects of TRPV1 signals are not known in bone tissues. The present study examined the effects of capsaicin, a natural TRPV1 ligand, on inflammatory bone resorption in vitro and in vivo. These results showed that the TRPV1 signal suppressed osteoclastic bone resorption associated with $\mathrm{PGE}_{2}$ production in vitro and attenuated inflammatory bone loss induced by LPS in vivo.

\section{Materials and Methods}

2.1. Materials and Animals. Capsaicin (Figure 1(a)) was obtained from Wako Pure Chemical Co. Ltd. (Tokyo, Japan). LPS (Escherichia coli O55:B5) was purchased from Becton Dickinson Co. Ltd. (Franklin lakes, NJ). PGE2 was obtained from Sigma-Aldrich Co. LLC (St Louis, MO). Newborn (2day-old and 5-day-old) and adult (6-week-old) $d d y$ mice were obtained from Japan SLC Inc. (Shizuoka, Japan). A PGE2 enzyme immunoassay (EIA) kit was purchased from Amersham Co. Ltd. (Aylesbury, UK). All other chemicals were of analytical grade.

2.2. Primary Mouse Osteoblastic Cells. Primary osteoblastic cells were isolated from newborn mouse calvariae after five routine sequential digestions with $0.1 \%$ collagenase (Wako Pure Chemical) and $0.2 \%$ dispase (Godo Shusei Co. Ltd, Tomakomai, Japan) as previously described [3]. Osteoblastic cells collected from fractions 2-4 were combined and cultured in $\alpha$-modified MEM ( $\alpha \mathrm{MEM})$ supplemented with $10 \%$ fetal calf serum (FCS) at $37^{\circ} \mathrm{C}$ under $5 \% \mathrm{CO}_{2}$ in air.

2.3. Osteoclast Formation. Mouse bone marrow cells were cocultured with the primary osteoblastic cells for 7 days. The cells adhering to the well surface were stained for tartrateresistant acid phosphatase (TRAP), and TRAP-positive multinucleated cells containing three or more nuclei per cell were counted as osteoclasts as previously described [10]. The concentrations of PGE2 in the cultured medium were determined using an enzyme immunoassay, as previously described [10].

2.4. Bone-Resorbing Activity in Organ Culture of Mouse Calvariae. Mouse calvariae were collected from 5-day-old mice and then were cultured for $24 \mathrm{~h}$ in BGJb medium containing $1 \mathrm{mg} / \mathrm{mL}$ bovine serum albumin (BSA). The calvaria was transferred into new medium, with or without LPS after
$24 \mathrm{~h}$, and then was cultured for 5 days at $37^{\circ} \mathrm{C}$ under $5 \%$ $\mathrm{CO}_{2}$ in air. The bone-resorbing activity was determined by measuring the concentration of calcium in the conditioned medium using a calcium kit (calcium $\mathrm{C}$ test; Wako Pure Chemical) as reported previously [2]. The bone-resorbing activity was expressed as an increase in medium calcium, which is consistent with the osteoclastic bone resorption as shown in the previous studies [2].

2.5. RT-PCR Analysis. Primary mouse osteoblastic cells were cultured for $24 \mathrm{~h}$ in $\alpha \mathrm{MEM}$ containing 1\% FCS then treated with LPS for $3 \mathrm{~h}$. The total RNA was extracted from mouse osteoblasts using the acid guanidium-phenol-chloroform method. cDNA was synthesized from $10 \mu \mathrm{g}$ of total RNA by reverse transcriptase (Superscript II Preamplification System; Invitrogen Life Technologies Co., Carlsbad, CA) and amplified using PCR. Primers for the mouse COX-1, COX-2, mPGES-1, and glyceraldehydes-3-phosphate dehydrogenase (GAPDH) genes were used in PCR as reported previously [4]. The PCR product was separated on a $1 \%$ agarose gel and stained with ethidium bromide. The signals were quantified using an image analyzer.

2.6. Inflammatory Bone Loss in Mice. Six-week-old mice were i.p. injected with LPS ( $10 \mathrm{mg} / \mathrm{kg}$ body weight) on days 0 and 4. The LPS was dissolved in PBS for injection. The mice in the control group were injected with PBS. Some mice were i.p. injected with capsaicin $(1 \mathrm{mg} / \mathrm{kg}$ body weight). The femurs were collected 8 days after the first injection of LPS or phosphate-buffered saline (PBS). For each group, 6-9 mice were used. The bone mineral density (BMD) of femurs was measured by dual X-ray absorptiometry (model DCS-600R; Aloka, Co. Ltd, Tokyo, Japan) as reported previously [4]. The bone mineral content of the femurs was closely correlated with the ash weight [4]. The BMD was calculated by dividing the bone mineral content of the measured area by the area.

2.7. Statistical Analysis. The data are expressed as the mean \pm SEM. The significant differences were analyzed using Student's $t$-test.

\section{Results}

LPS markedly induced osteoclast differentiation in cocultures of mouse bone marrow cells and osteoblasts on day 7 , while $30 \mu \mathrm{M}$ of capsaicin completely suppressed the osteoclast formation induced by LPS (Figure 1(b)). The conditioned coculture medium was examined to ELISA to determine the effects of capsaicin on $\mathrm{PGE}_{2}$ production induced by LPS. The level of $\mathrm{PGE}_{2}$ in the conditioned medium treated with LPS was higher than that of the control and was clearly suppressed by adding $30 \mu \mathrm{M}$ capsaicin in the coculture (Figure $1(\mathrm{c})$ ). Bone-resorbing activity was measured by the increase in calcium in the conditioned medium. LPS markedly induced bone-resorbing activity in calvarial cultures. The addition of $30 \mu \mathrm{M}$ capsaicin significantly suppressed the bone-resorbing activity induced by LPS (Figure 1(d)). These results indicate that capsaicin clearly 
<smiles>COc1cc(CNC(=O)CCCC/C=C/C(C)C)ccc1O</smiles>

(a)

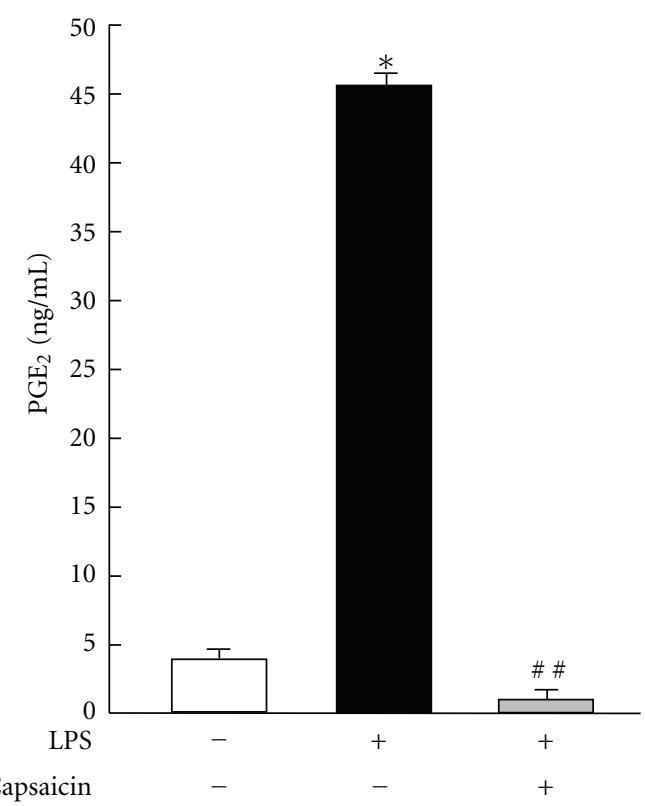

(c)

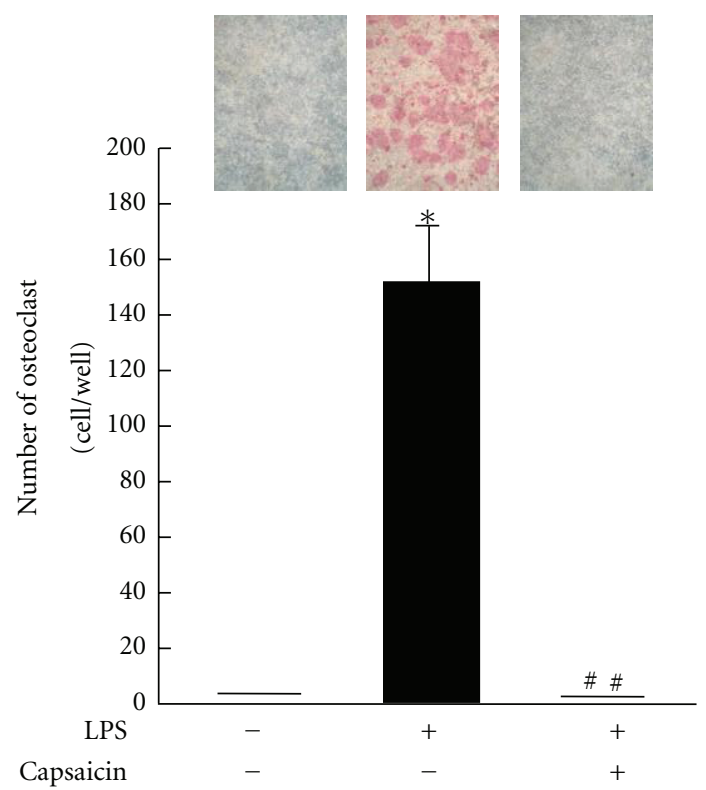

(b)

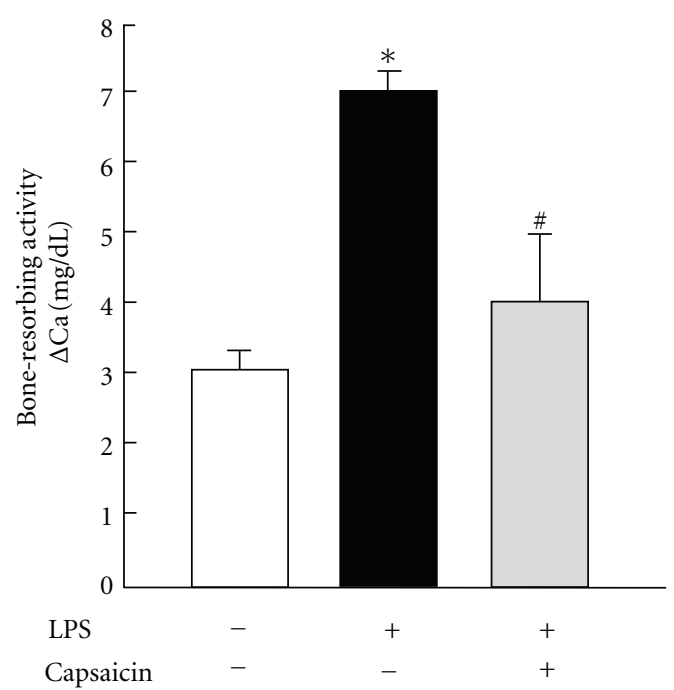

(d)

FIGURE 1: The effects of capsaicin on LPS-induced osteoclast formation and $\mathrm{PGE}_{2}$ production in cocultures of osteoblasts and bone marrow cells and on the bone-resorbing activity induced by LPS in mouse calvarial organ cultures. (a) Chemical structure of capsaicin. (b) Mouse bone marrow cells and osteoblastic cells were cocultured for 7 days with $30 \mu \mathrm{M}$ capsaicin in the presence of LPS ( $1 \mathrm{ng} / \mathrm{mL}$ ). The cells were stained for tartrate-resistant acid phosphatase (TRAP), a specific marker for osteoclasts, and the number of TRAP-positive multinucleated cells containing 3 or more nuclei was counted. The data are expressed as the mean \pm SEM of 4 independent wells. (c) The level of PGE was $_{2}$ measured by EIA using the conditioned medium of the cocultures. (d) Mouse calvariae were dissected in half and cultured with or without capsaicin $(30 \mu \mathrm{M})$ in the presence of LPS $(1 \mu \mathrm{g} / \mathrm{mL})$ for 5 days. The concentration of calcium in the medium was measured to calculate bone-resorbing activity. Data are expressed as the mean \pm SEM of 4 independent wells. A significant difference between the two groups is indicated, ${ }^{*} P<0.001$ versus control; ${ }^{\#} P<0.01$ versus LPS; ${ }^{\# \#} P<0.001$ versus LPS.

suppressed the osteoclastic bone resorption associated with inflammation in vitro.

$\mathrm{PGE}_{2}$ is mainly produced by osteoblasts in bone tissues. The expression of COX-2 and mPGES-1 is essential for
$\mathrm{PGE}_{2}$ production by osteoblasts treated with bone-resorbing cytokines such as IL-1 and LPS, and the PGE 2 production is essential for inflammatory bone resorption [4]. An RTPCR analysis showed the expression of COX-2 and mPGES-1 


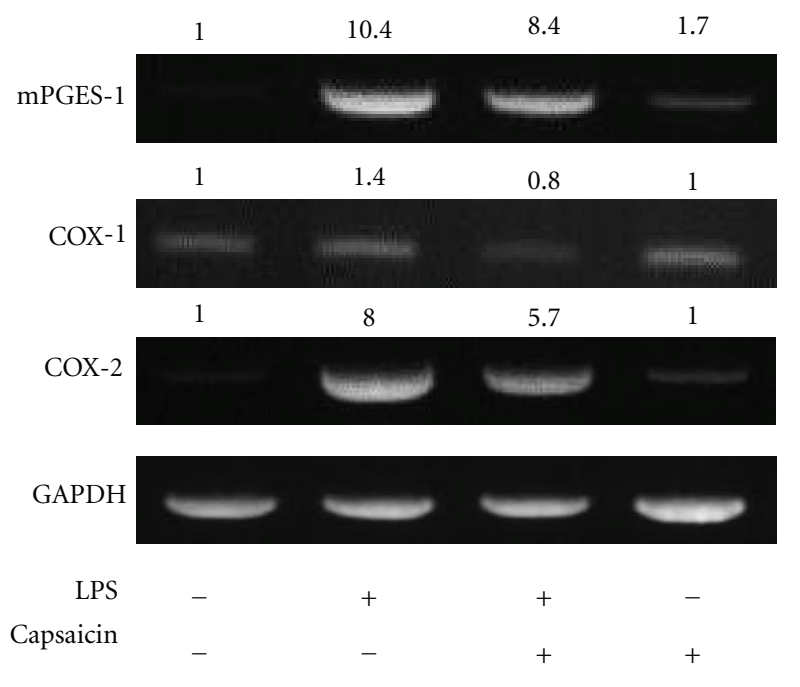

(a)

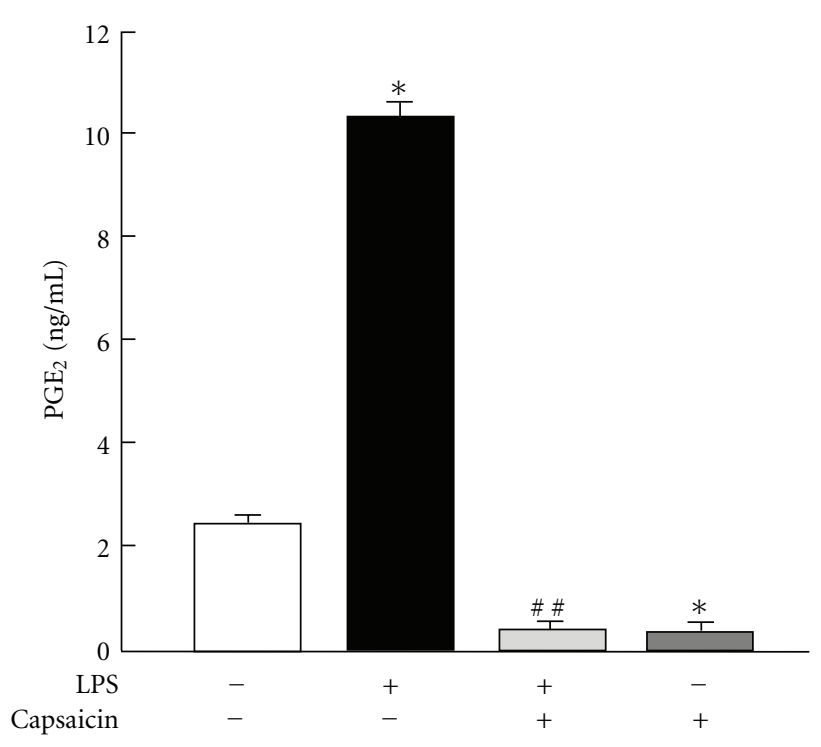

(b)

FIGURE 2: Effects of capsaicin on the production of $\mathrm{PGE}_{2}$ and the expression of COXs and mPGES-1 mRNAs in mouse primary osteoblasts. (a) Mouse osteoblasts were cultured with LPS $(1 \mathrm{ng} / \mathrm{mL})$ for $3 \mathrm{~h}$ with or without capsaicin $(30 \mu \mathrm{M})$. Total RNA was extracted, and the expression of COX-1, COX-2, and mPGES-1 mRNAs was detected using RT-PCR. The relative intensity of mRNA expression was calculated and noted in the upper of respective signal. (b) Osteoblasts were treated with $30 \mu \mathrm{M}$ capsaicin in the presence of LPS ( $1 \mathrm{ng} / \mathrm{mL}$ ) for $24 \mathrm{~h}$. The levels of $\mathrm{PGE}_{2}$ were measured using the conditioned medium. Data are expressed as the mean $\pm \mathrm{SEM}$ of 3 independent wells. A significant difference between the two groups is indicated, ${ }^{*} P<0.001$ versus control; ${ }^{\#} P<0.001$ versus LPS.

mRNAs to be markedly induced by LPS in mouse primary osteoblasts at $3 \mathrm{~h}$, and simultaneous addition of capsaicin clearly suppressed both the COX-2 and mPGES- 1 expression (Figure 2(a)). A low level COX-1 mRNA expression was detected in the osteoblasts, and this expression was not influenced by LPS or capsaicin. Capsaicin significantly suppressed LPS-induced $\mathrm{PGE}_{2}$ production in mouse primary osteoblast culture (Figure 2(b)).

LPS administration induces the severe loss of trabecular bone in distal femurs in mice, and mPGES-1 knockout mice are resistant to the bone loss induced by LPS [4]. The mice were i.p. injected with capsaicin with or without LPS, and the femurs were collected on day 8 for measurement of femoral BMDs. Distal femoral BMD was markedly suppressed by the treatment with LPS, and simultaneous treatment with capsaicin significantly restored the LPS-induced bone loss in mice (Figure 3). Capsaicin did not influence the femoral BMDs in the control mice without LPS.

\section{Discussion}

The present study showed that capsaicin, a TRPV1 ligand, suppresses LPS-induced osteoclastic bone resorption associated with inflammation by inhibiting $\mathrm{PGE}_{2}$ production by osteoblasts. $\mathrm{PGE}_{2}$ is a typical inducer of bone resorption associated with inflammation. We have reported that $\mathrm{PGE}_{2}$ binds to the EP4 receptor, one of the PGE receptor subtypes EP1-EP4, and induces the RANKL expression to stimulate bone resorption, using agonist and antagonist of EPs and respective EP-knockout mice $[2,11] . \mathrm{PGE}_{2}$ produced by osteoblasts binds to EP4 in osteoblasts and induces RANKL expression via EP4-mediated signals. LPS markedly induces the expression of COX-2 and mPGES-1 mRNAs in osteoblasts, and the $\mathrm{PGE}_{2}$ production is essential for RANKLdependent osteoclast formation [4]. The transcriptional regulation is important to understand the biological significance in target tissues because mPGES-1 is an inducible terminal enzyme associated with PGE2 biosynthesis. The mRNA expression of mPGES- 1 is coupled with COX-2, and the induction of COX-2 mRNA proceeded mPGES- 1 after adding LPS. The mouse COX-2 gene promoter possesses functional regulatory elements for NF- $\kappa \mathrm{B}$, NFIL-6, AP-1, and c/EBP $\alpha$ [12]. In contrast, the mouse mPGES- 1 gene promoter possesses AP-1 and c/EBP, but not NF- $\kappa$ B [13]. Capsaicin clearly suppressed the LPS-induced expression of COX-2 and mPGES- 1 mRNAs in osteoblasts in the present study. Further studies are needed to define the transcriptional regulation of COX-2 and mPGES-1 by capsaicin in osteoblasts.

Capsaicin, a typical ligand for TRPV1, is derived from chili peppers, which elicit a burning sensation through TRPV1 $[5,6]$. Mouse primary osteoblasts and osteoblast cell line MC3T3-E1 express TRPV1, but expression of TRPV1 mRNA is not detected in bone marrow macrophages, and that capsaicin suppresses IL-1-induced osteoclast differentiation in the cocultures of mouse bone marrow cells and osteoblasts [8]. In addition, both resiniferatoxin, a natural TRPV1 agonist, and olvanil, a synthetic TRPV1 agonist, suppress osteoclast formation induced by IL-1 in the cocultures (Miyaura and Inada; unpublished data). The present study 


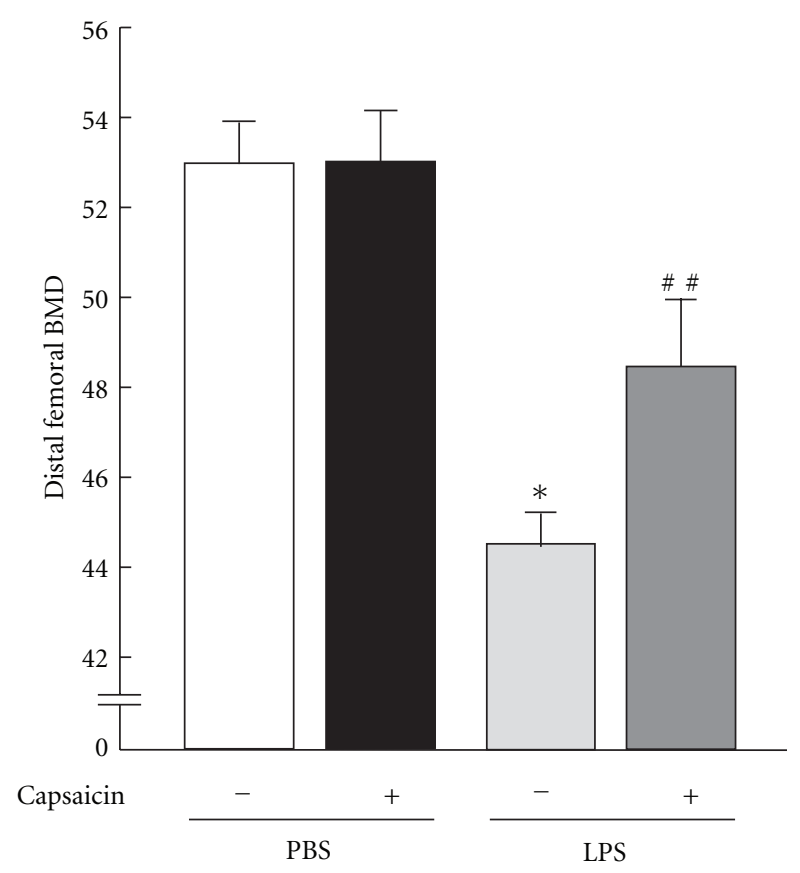

FIGURE 3: Effects of capsaicin on femoral bone mass in mice injected with LPS. The mice were injected with $(+)$ LPS $(10 \mathrm{mg} / \mathrm{kg}$ of body weight) or PBS (-) i.p. on days 0 and 4 with or without capsaicin ( $1 \mathrm{mg} / \mathrm{kg}$ of body weight). The femurs were collected on day 8 after the first injection to measure the bone mineral density (BMD). BMD of distal femurs was measured by dual X-ray absorptiometry (model DCS-600R; Aloka). Data are expressed as the mean \pm SEM of 6-9 mice. A significant difference between the two groups is indicated, ${ }^{*} P<0.001$ versus control; ${ }^{\#} P<0.001$ versus LPS.

found that capsaicin suppressed the $\mathrm{PGE}_{2}$ production induced by LPS in osteoblasts and suppressed LPS-induced osteoclast formation (Figures 1 and 2). These results indicate that osteoblasts are main target cells for TRPV1 ligand in bone tissues and regulate osteoclast differentiation associated with inflammation.

Chen et al. [14] reported that capsaicin acts on the macrophage cell line RAW264.7 to attenuate LPS-induced COX2 expression, but RAW 264.7 cells do not express TRPV1. On the other hand, Rossi et al. [9] have shown that human mature osteoclasts express TRPV1 isoforms and TRPV1 agonist enhances the expression and the activity of TRAP and Cathepsin K, two specific osteoclast biomarkers. The addition of capsaicin to bone marrow macrophages cultures in the presence of soluble RANKL and macrophage colony stimulating factor (M-CSF) does not influence osteoclast differentiation from macrophages (Miyaura and Inada; unpublished data). Idris et al. [15] have shown that capsazepine, a TRPV1 antagonist, acts on preosteoclasts and suppresses the differentiation into mature osteoclasts. Therefore, the roles of TRPV1 signals still remain controversial in macrophages, preosteoclasts, and mature osteoclasts.

The current study showed that capsaicin significantly restored inflammatory bone loss in the femur induced by LPS in mice (Figure 3). Therefore, TRPV1 ligands possess potential as clinical drugs targeting bone diseases associated with inflammatory bone resorption. Sancho et al. [16] reported that capsaicin has the potential to protect against inflammatory bowel diseases associated with severe inflammation, thus indicating the anti-inflammatory effects of TRPV1 signal in intestinal diseases. Idris et al. [15] reported that the TRPV1 antagonist capsazepine inhibits bone loss due to estrogen deficiency in ovariectomized (OVX) mice. However, the mechanisms of bone loss in OVX animals differ from that in LPS-treated animals accompanied with severe inflammation. In addition, Clark et al. [17] reported that TRPV4, a calcium permeable ion channel, possess chondroprotective role and deletion of TRPV4 gene resulted in severe osteoarthritis using TRPV4 knockout mice. Further studies using several animal models for bone and cartilage diseases are needed to define the possible roles of TRPVs signals in bone.

\section{References}

[1] L. G. Raisz, J. Y. Vanderhoek, and H. A. Simmons, "Prostaglandin synthesis by fetal rat bone in vitro: evidence for a role of prostacyclin," Prostaglandins, vol. 17, no. 6, pp. 905-914, 1979.

[2] C. Miyaura, M. Inada, T. Suzawa et al., "Impaired bone resorption to prostaglandin $\mathrm{E}_{2}$ in prostaglandin E receptor EP4knockout mice," Journal of Biological Chemistry, vol. 275, no. 26, pp. 19819-19823, 2000.

[3] Q. R. Chen, C. Miyaura, S. Higashi et al., "Activation of cytosolic phospholipase $\mathrm{A}_{2}$ by platelet-derived growth factor is essential for cyclooxygenase-2-dependent prostaglandin $\mathrm{E}_{2}$ synthesis in mouse osteoblasts cultured with interleukin-1," Journal of Biological Chemistry, vol. 272, no. 9, pp. 5952-5958, 1997.

[4] M. Inada, C. Matsumoto, S. Uematsu, S. Akira, and C. Miyaura, "Membrane-bound prostaglandin E synthase-1mediated prostaglandin $\mathrm{E}_{2}$ production by osteoblast plays a critical role in lipopolysaccharide-induced bone loss associated with inflammation," Journal of Immunology, vol. 177, no. 3, pp. 1879-1885, 2006.

[5] D. E. Clapham, "TRP channels as cellular sensors," Nature, vol. 426, no. 6966, pp. 517-524, 2003.

[6] M. J. Caterina, M. A. Schumacher, M. Tominaga, T. A. Rosen, J. D. Levine, and D. Julius, "The capsaicin receptor: a heatactivated ion channel in the pain pathway," Nature, vol. 389, no. 6653, pp. 816-824, 1997.

[7] J. B. Davis, J. Gray, M. J. Gunthorpe et al., "Vanilloid receptor1 is essential for inflammatory thermal hyperalgesia," Nature, vol. 405, no. 6783, pp. 183-187, 2000.

[8] M. Takita, M. Inada, and C. Miyaura, "Capsaicin, a ligand for vanilloid receptor-1, transduces suppressive signal for osteoclast differentiation in bone," Journal of Health Science, vol. 53, no. 2, pp. 240-244, 2007.

[9] F. Rossi, D. Siniscalco, L. Luongo et al., "The endovanilloid/endocannabinoid system in human osteoclasts: possible involvement in bone formation and resorption," Bone, vol. 44, no. 3, pp. 476-484, 2009.

[10] C. Miyaura, M. Inada, C. Matsumoto et al., "An essential role of cytosolic phospholipase A2 $\alpha$ in prostaglandin $\mathrm{E}_{2}$-mediated bone resorption associated with inflammation," Journal of Experimental Medicine, vol. 197, no. 10, pp. 1303-1310, 2003.

[11] T. Suzawa, C. Miyaura, M. Inada et al., "The role of prostaglandin $\mathrm{E}$ receptor subtypes (EP1, EP2, EP3, and EP4) in bone resorption: An analysis using specific agonists for the 
respective EPs," Endocrinology, vol. 141, no. 4, pp. 1554-1559, 2000.

[12] K. Yamamoto, T. Arakawa, N. Ueda, and S. Yamamoto, “Transcriptional roles of nuclear factor $\kappa \mathrm{B}$ and nuclear factorinterleukin- 6 in the tumor necrosis factor $\alpha$-dependent induction of cyclooxygenase-2 in MC3T3-E1 cells," Journal of Biological Chemistry, vol. 270, no. 52, pp. 31315-31320, 1995.

[13] H. Naraba, C. Yokoyama, N. Tago et al., "Transcriptional regulation of the membrane-associated prostaglandin $\mathrm{E}_{2}$ synthase gene: essential role of the transcription factor Egr-1," Journal of Biological Chemistry, vol. 277, no. 32, pp. 28601-28608, 2002.

[14] C. W. Chen, S. T. Lee, W. T. Wu, W. M. Fu, F. M. Ho, and W. W. Lin, "Signal transduction for inhibition of inducible nitric oxide synthase and cyclooxygenase- 2 induction by capsaicin and related analogs in macrophages," British Journal of Pharmacology, vol. 140, no. 6, pp. 1077-1087, 2003.

[15] A. I. Idris, E. Landao-Bassonga, and S. H. Ralston, "The TRPV1 ion channel antagonist capsazepine inhibits osteoclast and osteoblast differentiation in vitro and ovariectomy induced bone loss in vivo," Bone, vol. 46, no. 4, pp. 1089-1099, 2010.

[16] R. Sancho, C. Lucena, A. Macho et al., "Immunosuppressive activity of capsaicinoids: Capsiate derived from sweet peppers inhibits NF- $\kappa \mathrm{B}$ activation and is a potent antiinflammatory compound in vivo," European Journal of Immunology, vol. 32, no. 6, pp. 1753-1763, 2002.

[17] A. L. Clark, B. J. Votta, S. Kumar, W. Liedtke, and F. Guilak, "Chondroprotective role of the osmotically sensitive ion channel transient receptor potential vanilloid 4: age- and sex-dependent progression of osteoarthritis in Trpv4-deficient mice," Arthritis and Rheumatism, vol. 62, no. 10, pp. 2973-2983, 2010. 

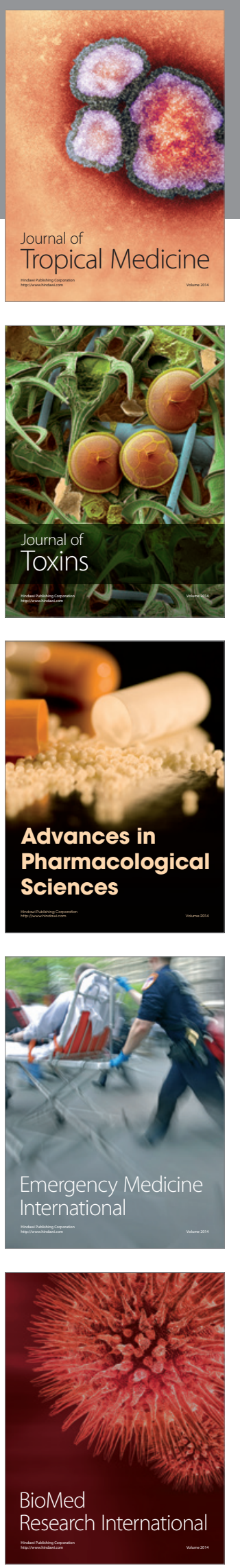
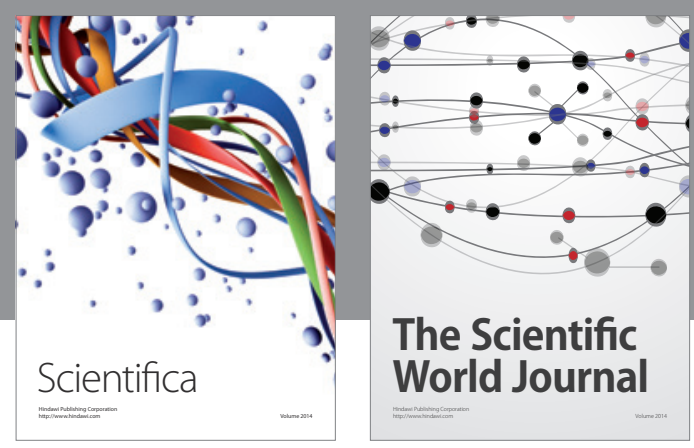

The Scientific World Journal
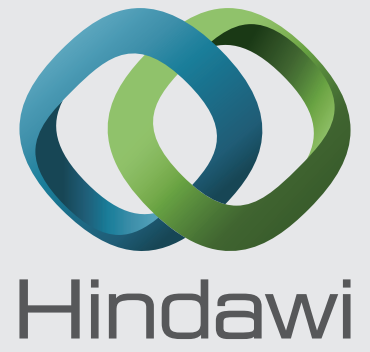

Submit your manuscripts at

http://www.hindawi.com
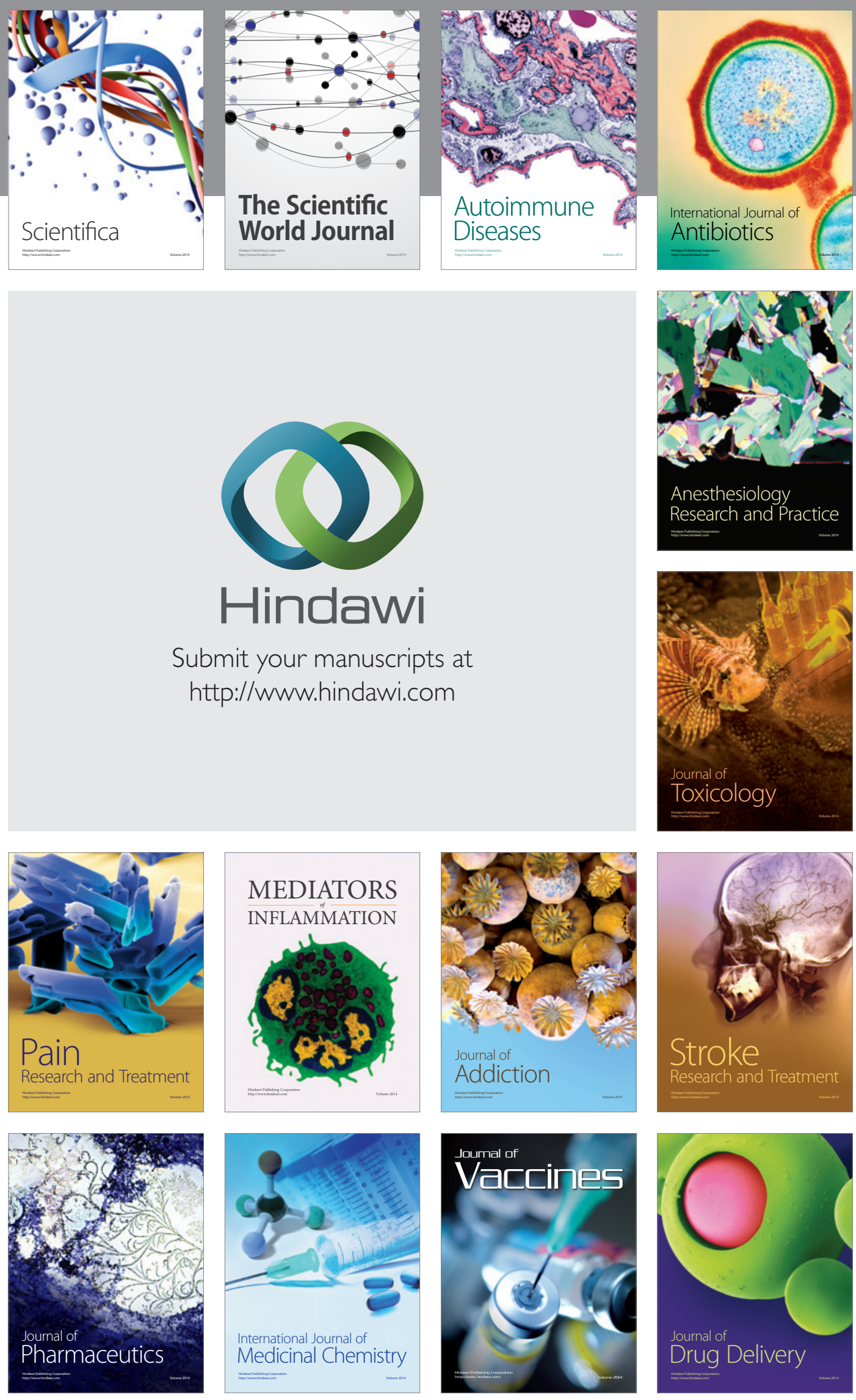